\title{
Inflamabilidade de espécies vegetais do cerrado
}

\author{
Núria Americo $^{\mathrm{a} *(\bullet}$, Maristela Lima Figueiredo Guimarães Epifanio ${ }^{\mathrm{a}}{ }^{\oplus}$, \\ Hygor Gomes de Almeida Sousa ${ }^{a}$, Priscila Bezerra de Souza ${ }^{a}$, \\ Francisca de Cássia Silva da Silva ${ }^{\mathrm{a}}{ }^{\oplus}$, Antonio Carlos Batista ${ }^{\mathrm{b}}$, Marcos Giongo ${ }^{\mathrm{a}}{ }^{\bullet}$ \\ a Universidade Federal do Tocantins, Brasil \\ b Universidade Federal do Paraná, Brasil \\ ${ }^{*}$ Autor correspondente (americonuria7@gmail.com)
}

\section{N F O}

\section{Keyworks}

index

flammability

Brazilian savannah

forest fires

\begin{abstract}
A B S T R A C T
Flammability of cerrado plant species.

Fire can cause damage and benefits to an ecosystem, and this relationship is influenced by two main factors: local weather conditions and fuel material characteristics. One of the main factors influencing the combustion process is the flammability of fuels, and its understanding is essential for the prevention of forest fires. Therefore, this study aimed to evaluate the flammability potential of Tabebuia aurea (Silva Manso) Benth. \& Hook.f. ex S.Moore, Pseudobombax grandiflorum (Cav.) A. Robyns, Dimorphandra mollis Benth, Chytraculia concinna (DC.) Kuntz, Eriotheca gracilipes (K. Schum.) A. Robyns and Qualea multiflora Mart. of cerrado sensu stricto and to indicate the species with the greatest potential to help prevent forest fires. The work was carried out at the Forest Research Laboratory at the Center for Environmental Monitoring and Fire Management (CeMAF) at the Federal University of Tocantins where flammability tests were conducted to evaluate ignition time, ignition frequency, combustion duration, combustion index and flammability value of the combustible materials, for each species studied, 50 firings were performed in a epiradiator, each burn consisted of $1 \pm 0.1 \mathrm{~g}$ of green combustible material. Based on the obtained data it was verified that among the studied species the one that showed the highest flammability index was Eriotheca gracilipes, on the other hand, Tabebuia aurea, Pseudobombax grandiflorum and Dimorphandra mollis species are considered to be weakly flammable, classifying them as potential for use in forest fire prevention.
\end{abstract}

\section{Palavras-chaves}

índice

inflamabilidade

Savana brasileira

incêndios florestais

\section{R E S U M O}

O uso do fogo pode ocasionar danos e benefícios a um ecossistema, sendo essa relação influenciada por dois fatores principais: condições meteorológicas locais e característica do material combustível. Um dos principais fatores que influenciam o processo de combustão é a inflamabilidade dos combustíveis, sendo considerada essencial a compreensão deste para a prevenção de incêndios florestais. Diante disso este trabalho objetivou avaliar o potencial de inflamabilidade de seis espécies do cerrado sensu stricto para auxiliar na prevenção de incêndios florestais, sendo elas: Tabebuia aurea (Silva Manso) Benth. \& Hook.f. ex S. Moore, Pseudobombax grandiflorum (Cav.) A. Robyns, Dimorphandra mollis Benth, Chytraculia concinna (DC.) Kuntz, Eriotheca gracilipes (K. Schum.) A. Robyns e Qualea multiflora Mart. O trabalho foi realizado no Laboratório de Pesquisas Florestais no Centro de Monitoramento Ambiental e Manejo do Fogo (CeMAF) na Universidade Federal do Tocantins. A partir dos testes de inflamabilidade foram avaliados o tempo para ignição, da frequência de ignição, duração do índice de combustão e valor de inflamabilidade dos materiais combustíveis, sendo que para cada espécie foram efetuadas 50 queimas em epirradiador, cada queima foi constituída de $1 \pm 0,1 \mathrm{~g}$ de material combustível verde. Com base nos resultados verificou-se que dentre as espécies estudadas a que demonstrou maior índice de inflamabilidade foi Eriotheca gracilipes, por outro lado, identificou-se que as espécies Tabebuia aurea, Pseudobombax grandiflorum e Dimorphandra mollis são consideradas como fracamente inflamáveis, classificando-as como potenciais para uso na prevenção de incêndios florestais. 


\section{INTRODUÇÃO}

O bioma Cerrado possui uma área de 2.036.448 $\mathrm{km}^{2}$, é classificado como o segundo maior bioma da América do Sul, possui uma extensão territorial muito relevante. (MMA, 2016). As vegetações deste bioma englobam formações florestais, savânicas e campestres, apresentando características como árvores com troncos tortuosos, cascas espessas, raízes profundas e algumas espécies perdem suas folhas como forma de reduzir a evapotranspiração no período de estiagem, ocasionando maior deposição de material combustível, esse aumento em associação com condições climáticas propicia a ocorrência de queimadas neste bioma (Ribeiro e Walter, 1998).

Devido à presença constante de fogo no Cerrado a vegetação adaptou-se, podendo atuar como agente de evolução para espécies florestais (Miranda et al., 2008). No entanto a alteração nos regimes naturais de queima compromete sua biodiversidade (Pivello, 2011). Diante da relação histórica do Cerrado com o fogo, o bioma evoluiu por meio de adaptações que o tornaram, em alguns casos até dependente da ação do fogo (Coutinho, 1990).

O uso do fogo pode gerar danos e benefícios a um ecossistema, sendo essa relação influenciada por dois fatores principais: condições meteorológicas locais e característica do material combustível (Hernando, 2009). O material combustível é todo o material orgânico presente na floresta e que está passível de ser queimado. A influência desse material no comportamento do fogo e por consequência na ação do fogo sobre o ecossistema, irá variar de acordo com suas características, tais como: distribuição, arranjo, tipologia, umidade, quantidade entre outros (Soares et al., 2017).

Um dos principais fatores que influenciam o processo de combustão é a inflamabilidade dos combustíveis florestais. A inflamabilidade está relacionada à propensão do material combustível em entrar em ignição, as propriedades que mantém o combustível queimando, a velocidade com que o combustível queima e a quantidade de combustível queimado (Hernando, 2009).

De acordo com Batista e Biondi (2009) existem muitas técnicas e medidas que podem ser implementadas para impedir ou diminuir os danos ocasionados pelos incêndios, dentre elas está conhecer as características fitofisionomicas da vegetação que compõe a floresta, e assim escolher a técnica mais eficaz para cada área. Considerando a escassez de pesquisas referentes a inflamabilidade de combustíveis florestais para favorecer o desenvolvimento de métodos de suspensão do fogo em casos de incêndios e tendo em vista que os demais fatores que influem no comportamento do fogo (clima e relevo) são incontroláveis, a compreensão das características de inflamabilidade de espécies do Cerrado pode ser essencial para a prevenção e controle de incêndios florestais neste Bioma.

Diante disso este trabalho objetivou avaliar a inflamabilidade das espécies Tabebuia aurea (Silva Manso) Benth. \& Hook.f. ex S.Moore, Pseudobombax grandiflorum (Cav.) A. Robyns, Dimorphandra mollis Benth, Chytraculia concinna (DC.) Kuntz, Eriotheca gracilipes (K. Schum.) A. Robyns and Qualea multiflora Mart., de grande ocorrência no cerrado sensu stricto, e indicar as espécies com maior potencial para auxiliar na prevenção de incêndios florestais.

\section{MATERIAL E MÉTODOS}

O trabalho foi realizado no Laboratório de Pesquisas Florestais no Centro de Monitoramento Ambiental e Manejo do Fogo (CeMAF) da Universidade Federal do Tocantins, campus de Gurupi, localizado em $11^{\circ} 46^{\prime} 25^{\prime \prime} \mathrm{S}$ e $49^{\circ} 02^{\prime} 05^{\prime \prime} \mathrm{W}$.

Foram coletados folhas e ramos das espécies Tabebuia aurea (caraiba), Pseudobombax grandiflorum (embiruçu), Dimorphandra mollis (faveira), Chytraculia concinna (guamirim), Eriotheca gracilipes (paineira-do-campo) e Qualea multiflora Mart. (pau-terra-liso) com diâmetro inferior a $0,7 \mathrm{~cm}$, classificados como material combustível fino (Soares et al., 2017).

Para determinar o teor de umidade do material combustível foi utilizada a equação 1 (Batista, 1990), sendo a massa seca obtida através de $200 \mathrm{~g}$ das amostras úmidas levadas à estufa por 48 horas a $75^{\circ} \mathrm{C}$.

$$
U=\left(\frac{M U-M S}{M S}\right) * 100
$$

Em que: $\mathrm{U}=$ teor de umidade do material (\%); MU = massa úmida do material no momento da coleta $(\mathrm{g})$; e $\mathrm{MS}=$ massa seca do material após estufa (g).

O teste de inflamabilidade foi conduzido conforme a metodologia proposta por Valette (1992), Petriccione (2006) e Kovalsyki et al. (2016). O material combustível foi submetido às avaliações até o tempo máximo de 2 horas após a sua coleta em campo, isso se deve em razão da necessidade de manter as condições das amostras mais naturais possíveis (Kovalsyki et al., 2016). O processo de queima do material combustível foi realizado em epirradiador em condições 
controladas, sem interferência de correntes de ar para assegurar a homogeneidade das condições para todas a queimas. Foi alocado, $4 \mathrm{~cm}$ acima do epirradiador, uma chama piloto com o objetivo de auxiliar na combustão inicial (Figura 01).

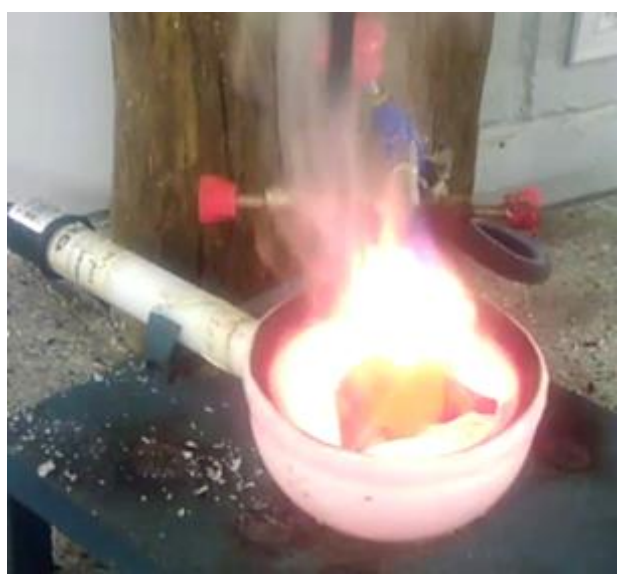

Figura 1 - Epirradiador durante a queima do material combustível.

Para cada espécie estudada, foram efetuadas 50 queimas. Cada queima foi constituída de $1 \pm 0,1 \mathrm{~g}$ de material combustível verde, obtido através de uma balança de precisão. Em todas as etapas foram utilizadas luvas de látex, como forma de evitar o contato direto com o material, para não influir nas propriedades do material. As características da combustão analisadas, de acordo com o proposto por Petriccione (2006), foram:

- Tempo para Ignição (TI): tempo percorrido até que se inicie a combustão do material combustível, em segundos;

- Frequência de Ignição (FI): número de vezes em que ocorre ignição, considerando um tempo de ignição de 60 segundos. Em queimas que ultrapasse este tempo serão consideradas como "queimas negativas";

- Duração da Combustão (DC): duração do tempo em que a chama permanece acesa;

- Índice de Combustão (IC): energia de combustão da queima, definida por meio da média do comprimento da chama e classificada segundo os índices dispostos na tabela 01.

- Valor de Inflamabilidade (VI): classificado mediante o FI e o TI (Tabela 02).

Tabela 1 - Índices do valor de combustão.

\begin{tabular}{ccc}
\hline Índice de combustão (IC) & Designação do IC & Comprimento da chama $(\mathrm{cm})$ \\
\hline ICI & Muito baixa & $<1$ \\
IC2 & Baixa & 1 a 3 \\
IC3 & Média & 4 a 7 \\
IC4 & Alta & 8 a 12 \\
IC5 & Muito alta & $<12$ \\
\hline
\end{tabular}

Fonte: Petriccione (2006).

Tabela 2 - Índices do valor de inflamabilidade.

\begin{tabular}{ccccccc}
\hline \multirow{2}{*}{ TI (s) } & \multicolumn{7}{c}{ FI } & & & \\
\cline { 2 - 6 } & $<25$ & $25-38$ & $39-41$ & $42-44$ & $45-47$ & $48-50$ \\
\hline$>32,5$ & 0 & 0 & 0 & 1 & 1 & 2 \\
$27,6-32,5$ & 0 & 0 & 1 & 1 & 2 & 2 \\
$22,6-27,5$ & 0 & 0 & 1 & 2 & 2 & 2 \\
$17,6-22,5$ & 1 & 1 & 2 & 2 & 3 & 3 \\
$12,6-17,5$ & 1 & 1 & 2 & 3 & 3 & 4 \\
$<12,6$ & 1 & 2 & 3 & 3 & 4 & 5 \\
\hline
\end{tabular}

Nota: FI - Frequência de Ignição e TI - Tempo para Ignição. Fonte: Petriccione, (2006).

O valor de inflamabilidade foi identificado através do número correspondente entre o tempo de ignição e a frequência de ignição, classificado em: $\mathrm{VI}=0$ (fracamente inflamável); VI $=1$ (pouco inflamável); VI = 2 (moderadamente inflamável); $\mathrm{VI}=3$ (inflamável); $\mathrm{VI}=4$ (altamente inflamável) ou VI $=5$ (extremamente inflamável) (Valette, 1992).

\section{RESULTADOS E DISCUSSÃO}

Na tabela 03 podem ser observados os resultados das queimas das espécies, seus percentuais de umidade e a frequência com que sofreram ignição. Nota-se que para os parâmetros da combustão a espécie que apresentou os melhores resultados foi $P$. grandiflorum com tempo médio para ignição de $60,83 \mathrm{~s}$, duração média de queima de 0,42 s e altura média de chama de $0,16 \mathrm{~cm}$, umidade do material 111,38 e frequência de ignição 2 . 
Tabela 3 - Resultados do teste de inflamabilidade para espécies estudadas.

\begin{tabular}{|c|c|c|c|c|c|}
\hline \multirow{2}{*}{ Espécies } & \multicolumn{3}{|c|}{ Médias } & \multirow{2}{*}{ U\%* } & \multirow{2}{*}{$\mathrm{FI}^{*}$} \\
\hline & TI $(\mathrm{s})^{*}$ & $\mathrm{DC}(\mathrm{s})^{*}$ & $\mathrm{HC}(\mathrm{cm})^{*}$ & & \\
\hline E. gracilipes & 17,62 & 16,55 & 16,42 & 52,38 & 2 \\
\hline T. aurea & 48,20 & 7,70 & 2,76 & 54,3 & 0 \\
\hline P. grandiflorum & 60,83 & 0,42 & 0,16 & 111,38 & 2 \\
\hline D. mollis & 45,58 & 2,69 & 3,96 & 103,39 & 40 \\
\hline C. concinna & 13,41 & 4,11 & 15,17 & 306,99 & 19 \\
\hline Q. multiflora & 21,13 & 7,83 & 16,16 & 217,17 & 27 \\
\hline
\end{tabular}

*TI (s) - Tempo para Ignição; DC (s) - Duração da Combustão; HC (cm) - Altura da Chama. U\% - Umidade do Material; FI - Frequência de Ignição.

A espécie E. gracilipes apresentou queimas com altas intensidades, com tempo médio de ignição $(17,62 \mathrm{~s})$ e altura média de chama $(16,42 \mathrm{~cm})$, assim como baixo teor de umidade $(52,38 \%)$. Segundo Hernando (2009), o teor de umidade dos materiais combustíveis está associado às variáveis que influenciam no comportamento do fogo, sobretudo o tempo de ignição e o comprimento inicial da chama. Por outro lado, a espécie $C$. concinna apresentou alto teor de umidade e parâmetros da queima elevados, necessitando de um estudo mais aprofundado sobre a espécie.

Kovalsyki et al. (2016) avaliando espécies para compor cortinas de segurança utilizou a espécie $P i$ nus taeda como controle e observou para a espécie um TI de 18,4 s, uma DC de 11,8 s e uma HC de $12,1 \mathrm{~cm}$ a uma umidade de $136 \%$ do material. Ao compararmos os resultados do autor supracitado aos obtidos neste trabalho para as espécies com umidades próximas, $P$. grandiflorum e $D$. mollis, observamos que os TI obtidos para essas espécies são muito superiores, 60,83 e $45,58 \mathrm{~s}$, respectivamente, enquanto que os valores de DC $(0,42$ e 2,69 s) e $\mathrm{HC}(0,16$ e $3,96 \mathrm{~cm})$ são inferiores, os menores dentre as espécies avaliadas.

Observando a tabela 04 verifica-se que as espécies $T$. aurea, $P$. grandiflorum e D. mollis obtiveram menores valores de índice de combustão $(2,1$ e 3, respectivamente), e o valor de inflamabilidade foi igual a 0 (fracamente inflamável), resultando assim nas espécies menos inflamáveis. Esse resultado pode estar relacionado com as variáveis tempo de ignição e altura de chama, pois para essas espécies obteve-se os menores valores de altura de chama e os mais longos tempos para ignição.

Tabela 4 - Índice de combustão e valor de inflamabilidade de seis espécies do Cerrado.

\begin{tabular}{|c|c|c|}
\hline Espécies & $\mathrm{IC}^{*}$ & $\mathrm{VI}^{*}$ \\
\hline E. gracilipes & 5 & 3 \\
\hline T. aurea & 2 & 0 \\
\hline P. grandiflorum & 1 & 0 \\
\hline D. mollis & 3 & 0 \\
\hline C. concinna & 5 & 1 \\
\hline Q. multiflora & 5 & 1 \\
\hline
\end{tabular}

* valores obtidos através de $200 \mathrm{~g}$ das amostras úmidas levadas à estufa por 48 horas a $75{ }^{\circ} \mathrm{C}$.

As espécies $C$. concinna e $Q$. multiflora obtiveram o resultado para valor de inflamabilidade igual a 1 (pouco inflamável), caracterizando-as como espécies com inflamabilidade muito baixa, devido ao tempo de ignição maior e a altura de chama com um valor elevado em relação às espécies que obtiveram a classificação do índice de inflamabilidade igual a 0 . Essas espécies apresentaram o maior teor de umidade dos materiais coletados, 306,99 e 217,17\%, respectivamente. Desta forma espécies de plantas com elevado teor de umidade tendem a serem menos inflamáveis por levarem mais tempo para entrar em ignição, isso se deve ao fato que comumente uma folha não inflama até que a maior parte de sua umidade seja perdida através do processo de evaporação (De Lillis et al., 2009.; Gill e Moore, 1996).

Batista e Biondi (2009) avaliando a inflamabilidade de Ligustrum lucidum, espécie largamente utilizada na arborização urbana, identificaram uma grande influência do teor de umidade do material nos parâmetros da combustão. Geralmente a umidade foliar chega a 300\% no período de folhação do vegetal e decrescendo para cerca de $50 \%$ devido ao processo de senescência foliar, e, assim como óleos essenciais voláteis, a umidade dificulta a 
absorção de energia pelo material durante o processo de combustão, influenciando diretamente na inflamabilidade (Soares e Batista, 2007; White e Zipperer, 2010).

A espécie com a maior inflamabilidade foi $E$. gracilipes com a classificação do índice de inflamabilidade igual a 3, caracterizando-a como inflamável, em razão do baixo tempo para ignição, a umidade baixa em relação às outras espécies, e o principal fator que contribuiu para esse índice de inflamabilidade alto: o tempo que a chama se manteve acesa.

Em levantamento de plantas de baixa inflamabilidade Souza e Vale (2019) observaram baixa inflamabilidade das espécies Vochysia thyrsoidea Pohl., Palicourea rigida Kunth., e Lavoisiera bergii Cogn. no cerrado do Distrito Federal, indicando-as para serem utilizadas em aceiros verdes. Kovalsyki et al. (2016) investigaram espécies para compor cortinas de segurança e observaram que as espécies Bougainvillea glabra Raddi. e Schinus terebinthifolius Choisy. foram as melhores, ambas com IC = 2 e VI $=0$, e ressaltaram esse resultado ao comparálas a espécie Pinus taeda que se mostrou inflamável de acordo com os testes.

Realizando uma comparação com os dados obtidos neste estudo verificou-se que as espécies $T$. $a u$ rea, $P$. grandiflorum e $D$. mollis ficaram com valores próximos as espécies com baixas inflamabilidades apontadas por esses trabalhos mencionados, isso se deve a vários fatores como, por exemplo, umidade do material, a sua composição química e espessura da folha.

\section{CONCLUSÕES}

Com base nos resultados deste estudo podemos verificar que dentre as espécies estudadas a que demonstrou maior índice de inflamabilidade foi a E. gracilipes. Por outro lado, identificou-se que as espécies $T$. aurea, $P$. grandiflorum e D. mollis são consideradas como fracamente inflamáveis, classificando-as como potenciais para uso na prevenção de incêndios florestais.

\section{REFERÊNCIAS BIBLIOGRÁFICAS}

Batista AC, Biondi D. Avaliação da inflamabilidade de $L i$ gustrum licidum Aiton (Oleaceae) para uso potencial em cortinas de segurança na região sul do Brasil. Revista Brasileira de Ciências Agrárias, v. 4, n. 4, p. 435-439, 2009.

Batista AC. Incêndios florestais. Recife: Imprensa Universitária da UFRPE, 115 p. 1990.

Coutinho LM. O cerrado e a ecologia do fogo. Ciência hoje, v. 12 , n. 68 , p. $23-30,1990$.

De Lillis M, Bianco PM, Loreto F. The influence of leaf water content and isoprenoids on flammability of some
Mediterranean woody species. Int J Wildland Fire, v. 18, p. 203-212, 2009.

Gill AM, Moore PHR. Ignitability of leaves of Australian plants. Canberra: CSIRO Plant Industry. 34 p. 1996.

Hernando CL. Combustibles forestales: inflamabilidad. La defensa contra incendios forestales, fundamentos y experiencias. McGraw-Hill, New York, p. 3-6, 2000.

Kovalsyki B, Takashina IC, Tres A, Tetto AF, Batista AC Inflamabilidade de espécies arbóreas para uso em cortinas de segurança na prevenção de incêndios florestais. Pesquisa Florestal Brasileira, v. 36, n. 88, p. 387-391, 2016.

MINISTÉRIO DO MEIO AMBIENTE (MMA). Consulta por: O Bioma Cerrado. Disponível em: <http://www.mma.gov.br/biomas/cerrado> Acesso em: 16 de abril. 2016.

Miranda HS, Bustamante MMC, Miranda AC. The Cerrados of Brazil: ecology and natural history of a neotropical savanna. The fire factor. p. 51-68, 2002.

Oliveira PS \& Marquis RJ. The cerrados of Brazil: ecology and natural history of a neotropical savanna. Columbia University Press, 2002.

Petriccione M. Infiammabilità della lettiera di diverse specie vegetali di ambiente mediterraneo. 2006. $48 \mathrm{f}$. Tese (Doutorado em Biologia Aplicada) - Dipartimento di Biologia Strutturale e Funzionale, Università Degli Studi Di Napoli Federico II, Napoli. 2006.

Pivello VR. The Use of Fire in the Cerrado and Amazonian Rainforests of Brazil: Past and Present. Fire Ecology, v. 7, 2011.

Ribeiro JF, Walter BMT. Fitofisionomias do bioma Cerrado. Embrapa Cerrados-Capítulo em livro científico (ALICE). p. 89-166, 1998.

SEPLAN/TO. Atlas do Tocantins: Subsídios ao planejamento da gestão territorial. 2002.

Soares RV, Batista AC, Tetto AF. Incêndios florestais: controle, efeitos e uso do fogo. Curitiba, $2^{\mathrm{a}}$ edição revisada, 255 p. 2017.

Souza MA, Vale, AT. Levantamento de plantas de baixa inflamabilidade em áreas queimadas de cerrado no Distrito Federal e análise das suas propriedades físicas. Universidade de Brasília, 2019.

Valette JC. Inflammabilities of Mediterranean species. Forest fire risk and management, v. 16719, p. 51-64, 1997.

White RH, Zipperer WC. Testing and classification of individual plants for fire behaviour: plant selection for the wildland-urban interface. International Journal of Wildland Fire, v. 19, n. 2, p. 213-227, 2010. 